\title{
JÓVENES QUE MIGRAN SOLOS. ACTORES Y ESCENARIOS FUERA DE LOS SISTEMAS DE PROTECCIÓN DE MENORES
}

\section{Youths who migrate alone. Actors and scenes outside of the children care systems}

Chabier Gimeno Monterde ${ }^{1}$

\section{Resumen}

Este artículo nace de una investigación más amplia en torno a los menores migrantes no acompañados que fueron tutelados por el Servicio de Protección de Menores del Gobierno de Aragón entre los años 2000 y 2010. Se ha accedido a los casi trescientos expedientes de tutela administrativa del periodo, que se han analizado estadísticamente. Los resultados muestran un colectivo de adolescentes magrebíes (78'5\%) y subsaharianos ( $\left.17^{\prime} 4 \%\right)$, del que apenas un 4'4 son niñas. Y para el que se han establecido varios periodos en el flujo migratorio, así como posibles pautas de su comportamiento frente a la acogida institucional, en relación con el sexo, el origen y la edad. Entre 2007 y 2012, las entrevistas a menores tutelados y extutelados, a técnicos de entidades públicas y privadas, así como la observación participante durante nuestra práctica profesional en proyectos educativos para jóvenes migrantes, han conformado el trabajo de campo en las tres provincias aragonesas. Entre otros análisis, concluimos que el itinerario de inserción social que los Servicios de Protección plantean a estos jóvenes a través de la tutela administrativa es sólo uno más de los múltiples itinerarios que, en paralelo, los menores recorren acompañados por actores a menudo ocultos a la Administración como los familiares en España y Europa y las redes de iguales; o en escenarios donde los Servicios Sociales tienen dificultades para actuar, como la infracción jurídica, la prostitución y las redes de explotación. Entender esta heterogeneidad de itinerarios creemos que evitará algunas situaciones graves de desprotección.

Palabras clave: menores no acompañados, migración irregular, transnacionalismo, protección de menores.

\begin{abstract}
This article stems from a larger research on unaccompanied migrant children who were protected by the Child Protection Service of the Government of Aragon between 2000 and 2010. The researcher obtained access to the almost three hundred cases under administrative supervision during that period. These cases were analyzed statistically. The results showed a group composed of young North Africans (78.5\%) and subSaharan youngters $(17.4 \%)$, of which barely a $4.4 \%$ are girls. Several periods in the migration flow and possible patterns of their behaviours in the host institution, in relation to sex, origin and age were established. Between 2007 and 2012, interviews with protected and former protected children and with technicians from public and private entities, as well as the participant observation during our professional practice in educational projects for young migrants defined the fieldwork in the three Aragonese provinces. Among other analyses, we concluded that the social integration schedule that Protection Services offers to these young people through administrative protection is just one of the many itineraries that these children go through in parallel: accompanied by actors often unknown to the Administration, such as relatives in Spain and Europe and peer networks; or in scenarios where Social Services find it difficult to intervene such as legal infringement, prostitution and exploitation networks. Understanding this heterogeneity of itineraries will avoid, we think, some serious situations of lack of protection.
\end{abstract}

Keywords: unaccompanied minors, irregular migration, transnationalism, child protection. 


\section{Introducción}

Los denominados «menores extranjeros no acompañados» son aquellos jóvenes menores de edad que migran solos e irregularmente de las áreas pobres del Planeta a las más ricas. La legislación internacional, al igual que la española, ampara su acogida en los sistemas de protección de menores hasta que alcanzan la edad adulta (Quiroga, 2005).

Recientemente, y tras la migración de miles de menores por esta vía alternativa a la migración adulta (Jiménez, 2011: 104), esta acogida institucionalizada es de nuevo objeto de debate. Hasta el punto de cuestionar si las Comunidades Autónomas no habrán recibido competencias explícitas e implícitas en la política migratoria española. Explícitamente se les reclamaría que protegiera a estos jóvenes, mientras implícitamente se les responsabilizaría de evitar el efecto llamada que dicha acogida supone (Gimeno, 2013: 146).

Diversos informes emitidos por instituciones públicas que tutelan a estos menores o que velan por sus derechos, se refieren a menudo a un grupo homogéneo de jóvenes que son «conscientes» de su estatus legal de desamparo, y que han emigrado a Europa "para ser atendidos» por las administraciones locales (Justicia de Aragón, 2007: 122). Sin embargo, aquí nos planteamos un grupo de menores mucho más heterogéneo. Pues entendemos que, más allá de los adolescentes acogidos por las instituciones, hay otras vías de migración que permanecen ocultas, por voluntad de sus actores o, en ocasiones, por negligencia del Estado. De manera que el itinerario estandarizado por los Servicios de Protección autonómicos para atender a los menores no acompañados es sólo uno de los recorridos por éstos.

A continuación queremos evidenciar, por un lado, la existencia de estos otros itinerarios migratorios que, aunque discurren en paralelo, en ocasiones se cruzan con el institucional. Y por otro, los conflictos técnicos y éticos que esta heterogeneidad provoca en el Trabajo Social.

\section{Material y metodología}

Este texto surge de una de las líneas de trabajo de la investigación doctoral «Como una bolisma. Menores que migran solos a Aragón $»^{2}$, desarrollada en las tres capitales provinciales aragonesas, donde estos menores han sido acogidos por el gobierno autónomo.

2. Dirigida por los profesores Carlos Gómez Bahillo y Aurora López Azcona, de la Universidad de Zaragoza 
Entre 2007 y 2012 hemos realizado un trabajo de campo que ha incluido dieciséis entrevistas semiestructuradas a jóvenes migrantes y trenta y nueve a técnicos de administraciones públicas o entidades privadas. En este periodo el autor, trabajador social, ha realizado también observación participante en los proyectos educativos en los que contactaba con estos menores, además de en otros espacios urbanos donde concurren jóvenes migrantes.

Nuestro análisis documental sobre esta migración ha incluido los informes anuales sobre menores realizados por el defensor del pueblo autonómico (Justicia de Aragón), así como otros emitidos por o para el Instituto Aragonés de Servicios Sociales (responsable del Servicio de Protección de Menores) y por entidades sociales que trabajan con jóvenes migrantes.

Y, finalmente, el IASS nos ha facilitado información anonimizada sobre los 298 expedientes de tutela administrativa de los menores no acompañados acogidos entre enero de 2000 y diciembre de 2010. Estos datos se han tratado estadísticamente, con el programa SPSS, y los resultados se publicarán próximamente por el gobierno autónomo ${ }^{3}$.

Tabla 1: Técnicas y variables

\begin{tabular}{|l|l|}
\hline Técnica & Variables \\
\hline Entrevistas semiestructuradas a menores & $\begin{array}{l}\text { Contexto de origen. Trayectoria } \\
\text { migratoria. Detección. Acogida } \\
\text { institucional. Tensiones en la acogida. } \\
\text { Emancipación. }\end{array}$ \\
\hline Entrevistas semiestructuradas a técnicos & idem \\
\hline Análisis estadístico de expedientes & $\begin{array}{l}\text { Datos demográficos. Inicio y cierre del } \\
\text { expediente. Notificante. Motivo del } \\
\text { cierre. Modalidad residencial. }\end{array}$ \\
\hline Observación participante & $\begin{array}{l}\text { Relaciones entre iguales, con jóvenes } \\
\text { nativos, con el Servicio de Protección y } \\
\text { otros técnicos. }\end{array}$ \\
\hline
\end{tabular}

\section{Itinerarios paralelos}

Aunque lejos de la realidad de Cataluña o Madrid, donde ha sido evidente la presencia de menores no acompañados que vivían en la calle (Quiroga, 2009:

3. Una breve descripción de los primeros resultados nos permite hablar de un colectivo de adolescentes, entre 15 y 17 años de edad, procedentes del Magreb en un 78'5 \% y del África Occidental en un $17^{\prime} 4 \%$. De entre ellos apenas un $4^{\prime} 4 \%$ son niñas. 
201), lo observado durante el comienzo de siglo por los profesionales del Trabajo Social entrevistados en Aragón permite intuir que, también en esta Comunidad, ha habido otras vías por donde los menores no acompañados han migrado, al margen de la acogida institucional.

Para explicar esta dualidad (protección/desprotección), que implica que los menores que migran solos atraviesan diversas circunstancias jurídicas hasta la mayoría de edad, Liliana Suárez (2006: 13) habla de un «itinerario intermitente»: que busca la regularización, al mismo tiempo que conservar la autonomía del menor frente a la Administración. Para esta antropóloga, el «uso meramente instrumental» de dicho sistema se corresponde con estrategias mixtas, no siempre preconcebidas, que dificultan la conducción de los menores hacia la estandarización que buscan los Sistemas de Protección de Menores, con los consiguientes conflictos con sus profesionales ${ }^{4}$.

Nuestra investigación propone llevar más lejos estos análisis, animando a los técnicos implicados a valorar si los menores que están fuera de la protección, o entran y salen de ésta, no estarían sino en una fase más de un itinerario de múltiples vías. Este planteamiento explicaría por qué tantos itinerarios paralelos al de la acogida incluyen, con frecuencia, la desinstitucionalización pasiva (fugas de los menores) ${ }^{5}$. Y, en cualquier caso, aclara por qué el periodo de institucionalización no es necesariamente la etapa definitiva de sus trayectorias migratorias.

Reforzando esta propuesta, durante la observación participante de la investigación hemos detectado diversos itinerarios migratorios que han estado al margen de o entrando y saliendo de la protección. Dadas las características de esta migración clandestina, existirán otros itinerarios que no nos habrán sido accesibles, ni como profesionales ni como investigadores ${ }^{6}$; pero, aun así, nos servirán para informarnos de la heterogeneidad de esta migración.

Así, al perfil del menor detectado por el Sistema de Protección y que llega a la mayoría de edad cumpliendo los objetivos de inserción que el itinerario estandarizado por los protocolos de acogida le marca, podemos añadir otros itinerarios observados:

4. Nuestro cuaderno de campo (2009-2010) señala la estrategia «dentro y fuera» de menores solos que hacen un uso instrumental de un recurso, donde se les apoya desde el área de Trabajo Social de acuerdo a su «victimización» (López Riopedre, 2011). Mientras, continúan su vida en la calle, que incluye infracciones frecuentes. Esta conjunción («y») es ajena al planteamiento excluyente del Trabajo Social ( «o») y la subvierte.

5. Por oposición a la desinstitucionalización activa (la repatriación al lugar de origen).

6. Insistiendo en una línea que vertebra nuestra metodología: reconocer que siempre hay más «socius» del que percibimos, y que este reconocimiento no nos impide «intuir» a través de lo que sí hemos podido percibir (Bergua, 2011: 199). 
a. Itinerario de menor magrebí que llega con pasaporte falso de adulto para ser acogido por la familia extensa en destino. La familia posteriormente rechaza su compromiso de acogida, por lo que el menor deambula itinerante por la península, «guiado» por compatriotas adultos. Su paso por la calle lo vincula ocasionalmente a la prostitución masculina. Llega a la edad adulta sin regularizar su residencia y con fuertes nexos con la infracción y el «lumpemproletariado».

b. Itinerario de menores subsaharianas que llegan a destino adoptadas por la familia extensa. Ésta las fuerza a la prostitución, por lo que piden ayuda y son derivadas al Sistema de Protección.

c. Itinerario de menores magrebís acogidos por su familia extensa (o nuclear) en destino. Ésta, conocedora del Sistema de Protección, insta al menor a presentarse para ser acogido por éste. Una vez llegado a la mayoría de edad, el menor vuelve a ser acogido por la misma red familiar.

d. Itinerario de menores magrebís que conocen del Sistema de Protección a través de las redes de iguales (jóvenes compatriotas, tutelados o no). Acceden al sistema por éstas y al terminar la tutela vuelven a integrarse en las mismas redes, que les continúan acompañando como adultos clandestinos o regulares.

e. Itinerario de menores magrebís que entran y salen de la protección mediante «fugas», constando varias fechas de apertura y cierre en sus expedientes de tutela. A la ausencia de un proyecto migratorio acorde a las expectativas del Sistema de Protección se une, a menudo, el contacto con las infracciones.

f. Itinerario de menores europeas del Este que llegan a destino tratadas ${ }^{7}$ por su familia o por una red mafiosa. Al detectarse su presencia ejerciendo la prostitución se les deriva al Sistema de Protección. Habitualmente las menores abandonan el centro de acogida en pocas horas y vuelven con los adultos que las prostituyen, cambiando de provincia de residencia. En pocas ocasiones, las menores completan el itinerario de protección.

7. La diferencia entre «trata»y «tráfico de personas» reside en que el «traficking» busca el lucro de las redes por cruzar las fronteras entre Estados, mientras la trata se basa en el engaño y en la explotación de las víctimas, dentro o fuera del Estado de origen (Defensor del Pueblo, 2012). 


\section{Actores y escenarios fuera de la protección}

Para llegar a entender la complejidad de estos itinerarios paralelos, los Sistemas de Protección necesitan reconocer que el itinerario lineal de sus protocolos, el que va del desamparo a la protección, no se ajusta a la realidad. Al contrario, la protección no es sino una más de las etapas posibles en la migración de menores solos. Y esto es así porque este movimiento migratorio abarca más allá en el tiempo (no sólo lo acotado por la mayoría o minoría de edad) y el espacio (no sólo lo delimitado territorialmente por un Servicio de Protección); así como en los actores intervinientes y los escenarios donde se desarrolla.

La presencia de actores migratorios no insertados en los protocolos del Sistema de Protección, como pueden ser las redes de iguales y la familia nuclear o extensa, ha sido una constante en la investigación, así como en el acervo profesional acumulado en nuestra praxis. Estos actores, junto a los escenarios que interseccionan con la acogida institucional, como son la infracción y la prostitución, deben ser analizados como elementos centrales en la configuración de los «otros itinerarios».

\subsection{Redes de iguales}

Si la migración de los menores llamados «no acompañados» tiene una característica, esa es la heterogeneidad y la inestabilidad (Gimeno, 2010: 57). La mayoría de los términos que intentan definirla, esto es, situarla en un contexto estable, acaban sucumbiendo a la realidad. Algo que ha sido reconocido por los profesionales del Trabajo Social sin apenas resistencia durante las entrevistas. Esto es evidente al hablar de menores «desamparados», «solos» o «no acompañados». Cuando en el discurso institucional ${ }^{8}$ y en las entrevistas con profesionales la presencia continua de actores migratorios que acompañan a muchos de los menores es algo explicitado sin rubor.

Las redes de iguales, esto es, los jóvenes compatriotas de los menores que migran, apoyan a éstos para llegar al destino, para permanecer en él (generalmente en el Sistema de Protección) y para seguir el viaje (a nuevos destinos o intentando establecerse al terminar la acogida institucional).

Al igual que quienes han estudiado esta migración (Suárez, 2006: 22), los profesionales del IASS son conscientes de este acompañamiento entre iguales ${ }^{9}$ y de que la lógica de las redes que apoyan e informan no hace sino reducir los

8. Véanse Ararteko (2005: 205 y 211) y Quiroga (2009: 160 y 204).

9. Las entrevistas a técnicos y otros profesionales se reseñan como P1, P2, etc. Las entrevistas a menores, como M1, M2, etc. 
riesgos y los costes personales del proyecto migratorio. De ahí que racionalicen la presencia de este actor ajeno a la acogida.

Se presentan ellos mismos, muchos vienen con la dirección aprendida (...) Vente ahora, que ahora hay plaza, se avisaban (...) Si, porque se lo pasan de uno a otro (P1).

Yo pienso que esto fue como un bulo, que entre ellos había una fama de que en [ciudad] era fácil conseguir la documentación, porque había menos afluencia de gente, y fue de no tener ninguno a tener un montón de gente. Entre ellos mismos decían, lo verbalizaban, aquí es una ciudad tranquila, es más fácil. (...) Es que yo tengo un amigo, que me ha dicho que aquí es más fácil conseguir los papeles y conseguir trabajo (P37).

Por lo que el aparente «no querer saber» de la Administración, que sí es consciente de que no hay tal soledad migratoria generalizada, responde sobre todo a la «invisibilización» de las redes de iguales. Las redes están, pero no son tangibles en términos burocráticos. Algo que en el juego de burla y astucia propio de los menores migrantes (Bergua, 2005: 44) es tenido en cuenta, al menos para «no declararse ganadores mientras están en el campo del equipo contrario».

En el COA [Centro de Observación y Acogida] encuentro gente conocida, pero sin decir que los conozco, si no dirán que nos están trayendo a gente (M10).

Las redes de iguales, al constituir un modelo de solidaridad edataria, tienen un papel relevante en muchos de los itinerarios migratorios observados, así como en casi todas las fases de los mismos. El momento y la forma en que participan en la trayectoria migratoria varía según el origen geográfico de los menores. En el caso magrebí, en las redes tienen mayor presencia las nuevas tecnologías. Y en el caso subsahariano el peso del vínculo local previo entre los menores es mayor.

Cuando las redes intervienen al inicio del proyecto migratorio, en origen, el boca a boca transmite entre los jóvenes la presencia de los corredores o vías institucionalizadas de llegar a Europa. O bien es Messenger o el teléfono el canal que traslada de destino a origen esa información entre iguales.

Sí, aquí [Aragón] hay centro de menores, puedes estudiar, puedes salir más adelante. No como aquí [Marruecos] con diploma y parado en casa... Sabía que iba a venir a un centro y nada más... Sólo sabia decir centro, centro, (M12).

Hablamos por Messenger.. Que si quieres venir, que están bien. Porque es normal tener miedo, pero cuando te cuentan que están bien,... Uno que llevaba ya un año, los demás seis meses, en pisos, que están bien ... Si no, no voy a venir (M10). 
Una vez iniciado el viaje, las redes intervienen especialmente cuando se llega al Estado de destino. Si el menor conocía la vía de la institucionalización, los iguales pueden, en ocasiones, hacer una primera acogida, orientando sobre los pasos para introducirse en el itinerario de la protección. Y si el menor no conocía previamente el Sistema de Protección, los iguales pueden aconsejarle sobre la idoneidad de esa vía para acceder a la regularización administrativa y otros objetivos de esta etapa migratoria.

Nos hemos encontrado gente de un centro [de menores] y nos han dicho que vayamos a la policía... Me llevó uno de un centro (M12).

Me dijeron que sin papeles era muy chungo, no podría trabajar, y me mandaron unos chavales al centro de menores de Zaragoza (M14).

Por último, estas mismas redes intervienen en la trayectoria migratoria cuando esta se dirige hacia nuevos destinos. Durante la etapa de la protección, a través de las nuevas tecnologías de la información, los iguales informan a los menores tutelados de las opciones existentes, tanto en otras Comunidades Autónomas como al otro lado de los Pirineos. De forma que, cuando la protección termina al llegar a la edad adulta o agotar los recursos institucionales para los nuevos adultos ${ }^{10}$, las redes transnacionales acogen a los ex-tutelados en Francia, Bélgica, País Vasco, Andalucía, etc. O bien en Aragón, compartiendo pisos alquilados entre varios ex-tutelados.

Si conoces a alguien es más fácil moverse a otro país (M8).

Los mismos chavales que han salido del centro y no tienen dinero se han juntado en una misma casa, compartiendo (P37).

En su papel de «colchón social» frente a la incertidumbre, las redes contienen en su interior una multiplicidad de opciones frente a la precariedad y el riesgo. En ellas cabe no sólo la coordinación transnacional, sino también la espontaneidad y, en no pocas ocasiones, la empatía con los perdedores frente al Sistema de Protección o la legislación de extranjería. Junto a estas reacciones ante la fragilidad de la protección, en varias entrevistas ha surgido también el fracaso como algo que las redes transmiten como un riesgo muy presente. De hecho, técnicos y menores han coincidido en señalar la presencia de ex-tutelados y de algún menor no detectado en infraviviendas o kharbas ${ }^{11}$. Último escalón residencial en el fracaso del proyecto migratorio.

10. Recursos de emancipación para ex-tutelados y servicios sociales que ofrecen alternativas residenciales.

11. Kharba o خ غربة es una palabra coloquial usada en todo Marruecos, que describe un sitio abandonado o destruido. 
Hay gente [extutelados] que está sin casa, los subo a mi piso [de acogida], si me ve el jefe, me dirá lárgate... Gente que ha estado en el albergue... olía mal ... hemos compartido cama... No me gusta que le pase eso a la gente (M1).

A buscarse la vida, durmiendo en la calle, en el río, debajo del puente (M3).

Hasta a vivir en casas de campo, como ocupas, hasta que les han pillado robando un bolso (P37).

\subsection{Familia de origen y en Europa}

En el caso de la familia, extensa o nuclear, estamos ante un actor al que podemos adscribir (casi) las mismas intervenciones que a las redes de iguales, pero que cuenta con otras variables propias y plantea otros «retos» al Sistema de Protección, que ha percibido también la debilidad de la categoría «no acompañado» cuando se usa para uniformizar el papel de la familia en esta migración. Como ejemplo, en los informes del Justicia de Aragón la presencia de las familias es algo evidente ${ }^{12}$, aunque en ellos no se perciba si no una parte de la diversidad de intervenciones familiares en la migración de los tutelados.

Analizando la intervención de las familias en las diferentes fases de la migración, su papel en el diseño del proyecto migratorio en origen es diferente al de las redes. En este caso nos encontramos, como señala abundantemente la literatura (Quiroga, 2005: 72), ante familias que pueden compartir o no el proyecto del menor, así como que pueden facilitar o dificultar el mismo. Por tanto, el discurso uniforme en torno a familias con «desapego» por los menores que migran está, como todo lo homogéneo, lejos de la realidad observada.

Algo similar ocurre cuando la familia intenta minimizar el riesgo migratorio desde el destino, bien desde otras Comunidades Autónomas o bien desde otros Estados europeos (como Francia o Italia), que pueden servir de etapa intermedia en su viaje hasta Aragón. La visión territorial de los Sistemas de Protección, de nuevo, no permite ver con distancia y claridad la dimensión transnacional de la familia, muy superior al marco estatal ${ }^{13}$. La mayor capacidad

12. «Apareciendo cada vez más menores inmigrantes marroquíes teóricamente no acompañados pero de los que se sospecha que sí lo están, que si bien sus padres permanecen en Marruecos aquí tienen familia (tíos, hermanos mayores...) y dada la imposibilidad de reagruparse, utilizan este recurso para la atención y cuidado de los menores y, a ser posible, su regularización, pervirtiendo así el sistema de protección pues se parte de la base de que el menor estará mejor con su familia que en un centro» (Justicia de Aragón, 2007: 122).

13. Las entrevistas recogen varios itinerarios con etapas europeas. M4 llega de Argelia a Murcia y de ahí a Francia, de donde viene a Zaragoza animado por otro compatriota tutelado. Otros menores han pedido a la investigación que no demos ninguna referencia («si apagas eso sí») que pueda ligarlos a su paso previo por Francia, donde estaban acogidos por la familia extensa. Todos ellos reflejan una movilidad que se adapta a la 
de las familias para situar la acogida institucional en un escenario global, donde el acceso a los servicios sociales se contempla dentro de las estrategias de migración transnacional, permite a éstas hacer un consumo estratégico de dichas acogidas, difícil de rechazar desde la lógica burocrática del desamparo. El ocultamiento y la complejidad del diseño migratorio, así como el acompañamiento consiguiente durante la tutela reflejan los múltiples matices de algo difícil de asumir desde un Sistema de Protección diseñado inicialmente para menores «regulares».

Mi hermano estaba aquí [España] y un día llamó a mi tío [Francia] y le contó eso... que un chico vino y lo tutelaron en un piso, le dieron los papeles y estudió... Me pasaron a mí, ¿si vienes, vas a estudiar...? Yo dije sí... Me llevaron a un autobús, que cogía pasajeros de Francia a Marruecos... con un hermano de amigo de mi hermano... Llegamos por la noche aquí [Zaragoza]... al día siguiente me acompañó un amigo de... estuvo en Francia también... Él me llevó a la comisaría, a la central... Yo tenía miedo... entendí que le dijo que vine debajo de un autobús... Si te pregunta cualquier cosa, tú dile que sí... cuântos años... Luego llegamos al COA (M13).

También hemos constatado la existencia de familias que, una vez el menor está en el Estado de destino, o bien «dicen no poder» o bien no pueden hacerse cargo de los menores, por su situación económica o administrativa como unidad familiar en precario, pasando el Servicio de Protección a ser una vía para asegurar o bien la estabilidad económica del joven o bien la regularización del mismo.

Estas cosas son bastante difíiles de hablar con ellos... Ya la madre llorando y tal, hijo mío, buena suerte, te doy los teléfonos de tus tíos, que están en España, habla con ellos,... Él llama a sus tíos, que estaban aquí, cogieron sus tíos y bajaron a buscarlo. Una vez aquí, los tíos creen que no pueden afrontarlo... y hablan con el IASS (P13).

Aunque en otras muchas ocasiones los servicios de acogida sí que acaban detectando la presencia de las familias en destino, especialmente al acabar la tutela con la mayoría de edad y pasar el menor a vivir con dicha familia extensa, posible interfaz de la familia nuclear (Justicia de Aragón, 2007: 147). En nuestro cuaderno de campo registramos en mayo de 2010: "[Menor] me enseña en el móvil las fotos de su hermano. Vive en [España]. Cuando salga del centro, ira con él a vivir. Él le dijo que viniera a un centro, porque tiene mujer e hijos y no puede pagar sus gastos».

legislación durante el itinerario, igual que se adapta a los cambios de ésta (Jiménez, 2011: 279). 
Aun así, el Sistema de Protección sigue sin poder asumir al «consumidor de» la acogida (Gimeno, 2010: 66) en que se convierte el menor apoyado por la familia. Esta idea, anteriormente citada, de «perversión» de la tutela al sustituir ésta a la familia, se expresa en los informes institucionales junto a la clave de esta trayectoria migratoria: la imposibilidad de reagruparse familiarmente, que ha generado estrategias familiares híbridas, donde la subversión del Orden migratorio supera lo admisible por éste (Gimeno, 2011: 859).

Ahora bien, mientras que las redes de iguales eran «invisibles» burocráticamente, el Sistema de Protección sí que ha visualizado a algunas familias, lo que le ha permitido reposicionarse. $\mathrm{Y}$ esta es una variable que diferencia claramente familias e iguales: la invisibilidad de las familias es más frágil. La presencia de las mismas es algo que la Administración rastrea e intenta detectar desde el comienzo mismo del itinerario de acogida. Esta praxis es una reacción frente a la internacionalización del uso de la protección, pero también una herramienta de control sobre el menor, como hemos observado en los menores infractores. Aun así, durante la investigación no hemos detectado acciones de represalia frente a las familias nucleares en destino que utilizan la protección, algo que se ha empezado a producir en otras Comunidades Autónomas con mayor volumen de migración de menores ${ }^{14}$.

Se pasaron un montón de tiempo pidiendo que les dé el número de teléfono de mi padre, y no lo consiguieron (M13).

[Servicio de Protección de Menores] ha hablado con mi familia. Yo no he dicho nada, cuando acabe esto prefiero decirles que yo no soy ese cabrón que dicen [que está en centro de reforma]... Ha hablado un traductor y la coordinadora, no estaba yo delante, han hablado con mi hermano, por suerte, no lo sabe mi madre,.... ¿Por qué han hablado?, ¿para joderme? La familia saben que estoy aquí y estoy haciendo papeles,... no saben lo que está pasando (M12).

Otra variable que diferencia a las redes y a las familias como actores transnacionales es la capacidad evaluadora de la migración de los menores que éstas tienen. La familia está presente casi siempre, se encuentre en destino o en origen. Para los profesionales es la conciencia (o superego) del menor, en unas ocasiones, o la cogestora migratoria, en otras. En cualquier caso, la intervención en clave de evaluación es una constante en el discurso de la mayoría de los menores entrevistados.

14. «Andalucía detecta casos de niños marroquíes en falso desamparo», en http://elpais. com/diario/2011/10/27/sociedad/1319666403_850215.html. 
Sí, claro... Depende, cada semana, cada día, depende,... Ellos quieren verte bien, con tus papeles, con tu todo, sin problemas, con tu familia, quieren ver sólo bien. Te quieren ver mejor que ellos (M3).

Y, finalmente, hemos de plasmar la relación que ha existido entre la protección como sistema y la familia como actor visibilizado por éste. En este caso, la realidad de la gestión de esta migración desde el Sistema de Protección se define por lo que no es: no es transnacional. Frente a las propuestas teóricas (Esteban, 2010) o prácticas (Manzani, 2012) que preconizan una protección que cuente con las familias en origen, la praxis de acogida en Aragón, como en otras Comunidades, ha mantenido una relación instrumental con las familias de los menores. Esto es, se ha relacionado con las mismas para gestionar la regularización del menor, en los casos en que éste se adecuaba al perfil del itinerario estandarizado, o para influir en su «uso» del sistema ${ }^{15}$.

Así, los principales protagonistas del contacto del sistema de acogida con las familias han sido los propios menores. El Servicio de Protección ha asumido universalmente el derecho al contacto periódico, generalmente por teléfono ${ }^{16}$, del menor con su familia nuclear; asignando una dotación económica a tal efecto, que no ha sido retirada en función de sanciones u otras incidencias. Y, al mismo tiempo, ha externalizado a los menores la responsabilidad de aligerar los trámites de documentación y otros tendentes a su regularización. Si bien desde algunos recursos se ha trabajado en estas tareas, la inexistencia de enlaces estables en origen ha puesto en manos del menor gran parte de la responsabilidad de transmitir a su familia la información sobre los trámites precisos en el Estado originario.

¿Por qué no se ha realizado esta coordinación transnacional? Lo evidente es que existe un discurso institucional sobre el «efecto llamada» que estas buenas prácticas implicarían (Gimeno, 2013: 144). Asumiendo así algunas praxis de Trabajo Social con menores solos un nacionalismo metodológico claramente contradictorio con su ética profesional.

15. A este «uso» y a sus diversas variantes se refiere el Trabajo Social como «comportamiento», partiendo de un asistencialismo opuesto al menor como «consumidor de» la protección.

16. "Cuando vienen se les da una tarjeta para llamar a su casa,... Se recarga una vez al mes,... Depende de cómo están llaman o no» (P6). También hay ocasiones en que los menores utilizan los medios telemáticos del centro de acogida para contactar con sus familias: «O por internet, Messenger, con voz y con imagen, te presentan, mira mi madre, mi hermano, desde su propia casa o desde casa del vecino» (P36). 


\subsection{Escenarios fuera de la Protección}

Además de estos actores externos al Sistema de Protección, los trabajadores sociales asumimos que hay, al menos, dos escenarios con los que no sabemos, no podemos o no queremos intervenir: la infracción jurídica y las redes de explotación y/o prostitución.

En el caso de la infracción, la investigación ha desarrollado más ampliamente el análisis de ésta en otro texto ${ }^{17}$, por lo que aquí sólo insistiremos en que evidencia una tendencia a la desinstitucionalización, un rechazo a los itinerarios estandarizados, que no coinciden con las expectativas migratorias de algunos menores solos. La infracción es, en definitiva, una confrontación más entre la homogeneidad de resultados esperada por el Sistema de Protección y el imaginario migratorio de los menores solos. El imaginario de unos jóvenes globales que, aun dentro de la acogida institucional, ansían ser Primer Mundo (consumismo, hedonismo, etc), desarrollando para ello una heterogeneidad de procedimientos, unas veces dentro y otras fuera de la ley.

El otro escenario al margen de la protección es el transitado por los menores, especialmente, las menores que llegan a Aragón mal acompañadas por redes de explotación, dirigidas por adultos. Dentro o al margen de estas redes, la prostitución suele estar vinculada a la mayoría de estas trayectorias migratorias, diferenciadas para niños ${ }^{18} \mathrm{y}$ para niñas.

Se trata de los itinerarios menos conocidos por el Trabajo Social, que hemos explorado a través de las entrevistas a profesionales, especialmente de entidades sociales que atienden específicamente a trabajadoras sexuales. En estas entrevistas se señala la presencia de menores prostituidas, que provienen casi siempre del África Subsahariana y de Europa del Este ${ }^{19}$. En algunos casos, las menores subsaharianas están vinculadas a la trata de personas, pero aquí bajo redes familiares: menores ecuatoguineanas tratadas mediante «adopciones» que se han usado para burlar los controles migratorios. Algunas de estas menores han llegado a la edad adulta tras una trayectoria migratoria de invisibilización durante varios años.

Hace poco nos vino una chica con dieciocho, nigeriana, que había estado desde menor, en un piso por [Casco Viejo], lo llevaba una senegalesa (P11).

17. «Menores solos e infracción en Aragón» (Gimeno, 2012).

18. Los casos conocidos por este investigador de menores que se prostituyen, habitualmente fuera de redes de explotación, coinciden en su perfil con lo descrito por Ainhoa Rodríguez (2008).

19. La investigación de Ariadna Alonso (2008 y 2010) sobre menores del Este europeo prostituidas en Cataluña es una buena introducción a este escenario. 
En el caso de las europeas, casi siempre rumanas, aun habiendo más expedientes de protección, al convertirse este siglo en miembros de la Unión Europea las pautas migratorias son diferentes. El paso de los menores rumanos y rumanas por los centros de primera acogida ha sido siempre fugaz (Justicia de Aragón, 2008: 82), siguiendo una pauta extendida por nuestro entorno europeo (Senovilla, 2007: 49; TdH, 2009: 31). Lo más grave es que, muy a menudo, las menores del Este vinculadas a la prostitución se fugan de los centros o son recuperadas de éstos por redes familiares o mafiosas que las reintegran de nuevo en la actividad del comercio sexual. En estos casos, los técnicos públicos y privados coinciden en la inadecuación de las intervenciones que se desarrollan con estas menores, que deberían ser acogidas como víctimas de trata, eliminando el contacto con su «familia extensa» (Alonso, 2008: 142).

\section{Discusión}

A la vista de las estrategias migratorias presentadas, podemos concluir que las Comunidades Autónomas han recibido competencias explícitas e implícitas en la política migratoria española sobre menores no acompañados. Explícitamente se les reclama que los protejan, mientras implícitamente se les responsabiliza de evitar el efecto llamada que dicha acogida supone.

Con todo, los intentos de abordar la presencia de otros actores y escenarios, desde una acogida institucional posicionada como herramienta de control migratorio, se encuentran siempre con nuevas estrategias que los desbordan, generando nuevos itinerarios paralelos al de la protección. Por lo que parece necesario que los Servicios de Protección de Menores implementen praxis que, a través de la mediación, abarquen el entorno social transnacional de estos adolescentes.

Y, por último, el escenario reseñado de prostitución de menores nos invita a debatir como técnicos sobre la necesidad y urgencia de la modificación de algunas prácticas administrativas en la detección y acogida de las menores solas. Especialmente para evitar la fuga de los centros, presionadas por unas redes que las desplazan contra su voluntad por la geografía del Estado.

\section{Bibliografía}

Alonso, A. (2008). En terra de ningú. Una aproximació a la prostitució en els projectes migratoris de menors no acompanyades de l'Europa de l'Est a Catalunya. Tesina de Máster, Universitat Autònoma de Barcelona.

Alonso, A. (2010). En tierra de nadie. Migración y prostitución entre adolescentes no acompañadas de Europa del Este en Cataluña. Educación Social, 45, 65-77. 
ARARTEKo (2005). Situación de los menores extranjeros no acompañados en la CAPV. Vitoria: Ararteko.

Bergua, J.A. (2005). Lo social instituyente y la imaginación. Culturales, 1, 29-56. BERGUA, J.A. (2007). Lo social instituyente. Materiales para una sociología no clásica. Zaragoza: Prensas Universitarias.

Bergua, J.A. (2011). Estilos de la investigación social. Técnicas, epistemología, algo de anarquía y una pizca de sociosofía. Zaragoza: Prensas Universitarias.

Defensor del Pueblo (2012). Trata de seres humanos en España: víctimas invisibles. Madrid: Defensor del Pueblo.

Esteban, G. (2010). Propuestas para la creación de un sistema transnacional de protección de menores (entre España y Marruecos, en el marco de la Estrategia Europea sobre los Derechos de la Infancia). Propuestas para la actuación conjunta hispano-marroquí en el ámbito de la infancia: Colección Estudios e Informes sobre Inmigración $n^{\circ}$ 2. Granada: Editorial Comares.

GIMENO, Ch. (2010). Menores extranjeros no acompañados: una cuestión compleja para las políticas públicas y sociales. Revista de Derecho Migratorio y Extranjería, 25, 55-72.

GIMENO, Ch (2011). El orden y la gente: los menores migrantes no acompañados. En S. Giménez, y A. García, (coord.), Innovaciones en la sociedad del riesgo. Asociación Castellano Manchega de Sociología.

GIMENO, Ch (2012). Menores que migran solos e infracción en Aragón. Revista de Derecho Migratorio y Extranjería, 29, 263-283.

GIMENO, Ch. (2013). Expectativas de acogida en el imaginario de los menores que migran solos. REID, 9, 142-158.

JiMÉNEZ, M. (2011). Intrusos en la fortaleza. Menores marroquíes migrantes en la Frontera Sur de Europa. Tesis doctoral, Universidad Autónoma de Madrid.

Justicia de Aragón (2000 a 2010). Informes sobre la situación de los menores en Aragón. Zaragoza: Justicia de Aragón.

LÓPEZ RIOPEDRE, J. (2011, octubre). El proceso de victimización de la trabajadora sexual migrante. Comunicación en la International Conference on Negotiating Identity in Migration Processes. Madrid: CSIC. Pendiente de publicación.

MANZANI, L. et alter (2012, abril). La medicación transnacional para la mejora de la intervención socioeducativa con menores y jóvenes extranjeros sin referente familiar adulto en las sociedades receptoras. Póster en el Congreso sobre las Migraciones Internacionales en España. Bilbao.

Quiroga, V. (coord.) (2005) Rutas de pequeños sueños. Los menores migrantes no acompañados en Europa [Informe comparativo]. Barcelona: Fundación Pere Tarrés.

Quiroga, V. (coord.) (2009), Somnis de butxaca. Nois i noies menors migrants no acompanyats a Catalunya. Barcelona: Fundació Jaume Bofill. 
RODRíguez, A. (2008). Reacciones y relaciones de menores y jóvenes marroquíes ante la protección y la exclusión. E-migrinter, 2. Universidad de Poitiers (Francia).

SEnovilla, D. (2007). Situación y tratamiento de los menores extranjeros no acompañados en Europa. Bruselas: Observatorio Internacional de Justicia Juvenil.

SuÁREZ, L. (2006). Un nuevo actor migratorio: jóvenes, rutas y ritos juveniles transnacionales. En F. Checa, A. Arjona y J.C. Checa (Eds.), Menores tras la frontera. Otra inmigración que aguarda. Barcelona: Icaria.

TERRE DES HOMMES - TDH (2009). DisAPPEARING, departing, running away. A surfeit of children in Europe? Laussane (Suiza): TdH.

ANTECEDENTES DE DIFUSIÓN

Presentado un avance como comunicación a la International Conference Unprotected unaccompanied children in Europe: Which reasons lead to their lack of protection? Poitiers, Francia, celebrado el 22-24 de octubre de 2012.

Alternativas, 20, 2013, pp. 41-56 - ISSN 1133-0473 - DOI: 10.14198/ALTERN2013.20.03 